\title{
LA PESQUERÍA ORNAMENTAL DE LA ARAHUANA Osteoglossum bicirrhosum (OSTEOGLOSSIDAE) EN LORETO, PERÚ Y POSIBILIDADES DE SU CULTIVO
}

\author{
Fernando Alcántara Bocanegra" ${ }^{1 *}$, Fred William Chu-Koo', Carlos Alberto Chávez Veintemilla', Salvador \\ Tello Martín', Karin Cristina Bances Chávez ${ }^{2}$, Marco Antonio Torrejón Meza ${ }^{3}$, Jorge Luís Gómez \\ Noriega ${ }^{4} \&$ Javier Noriega Murrieta
}

\begin{abstract}
RESUMEN
La arahuana Osteoglossum bicirrhosum (Osteoglossidae) es un pez de cuerpo grande, de color plateado, que en el área de Loreto, Perú se comercializa como pez ornamental al estado de larva. En este artículo se presenta un análisis sobre las zonas y épocas de pesca, los volúmenes de comercialización y las posibilidades del cultivo de la arahuana, en la región Loreto (Perú). La extracción de las larvas de esta especie se realiza principalmente en el sistema del río Ucayali y la Reserva Nacional Pacaya Samiria en donde operan doce unidades de pesca comunitaria que, durante el año 2005 han capturado 80,000 larvas por un valor de 80,000 dólares americanos. La reproducción de la arahuana es estacional, con mayor intensidad entre los meses de diciembre y enero, en aparente correlación con la creciente de las aguas. Cada hembra desova de 100 a 300 huevos, que el macho incuba en la boca, hasta que se convierten en pequeños alevinos. Los niveles de captura y demanda de larvas de arahuana se han incrementado durante los últimos quince años aumentando la presión de pesca sobre este importante recurso que significa un nivel de ingreso anual de 600,000 a 1'000,000 de dólares americanos, por lo cual, se propone establecer programas de cultivo en estanques, debido a su reproducción natural en estos ambientes.
\end{abstract}

PALABRAS CLAVE: Arahuana Osteoglossum bicirrhosum, pesquería, cultivo.

\section{THE ORNAMENTAL FISHERY OF AROWANA Osteoglossum bicirrhosum (OSTEOGLOSSIDAAE) IN LORETO, PERU AND FEASIBILITY OF ITS CULTURE}

\begin{abstract}
The silver Arowana Osteoglossum bicirrhosum (Osteoglossidae) is a large fish whose larvae are being traded as a valuable ornamental fish in the Loreto region (Peru). The goal of this article is to provide a brief analysis of the fisheries, trading and feasibility of culturing this fish in the Loreto region (Peru). Larvae fisheries is practiced mainly in the Ucayali river system and into the Pacaya Samiria National Reserve where twelve community fisheries units operate legally and were the responsible of capturing 80,000 larvae from the nature making a profit of 80,000 American dollars in 2005. Arowana reproduction is seasonal with major intensity between December and January which apparently correlates to the raising water period. Each female Arowana can spawn between 100 to 300 eggs which will be entirely protected into the male's mouth until they become larvae. Arowana trade had increased in the last fifteen years in the Peruvian Amazon moving up the fishery pressure on this important ornamental resource and meaning an annual income of 600,000 to 1 000,000 USD for local fish traders. Therefore, it is extremely necessary to promote the culture of this important fish due to its ability of spawning naturally in terrestrial ponds if we want to make this activity sustainable.
\end{abstract}

KEYWORDS: Arowana, Osteoglossum bicirrhosum, fisheries, fish culture.

1 Instituto de Investigaciones de la Amazonía Peruana. Programa de Ecosistemas Acuáticos. Centro de Investigaciones Quistococha. Av. Abelardo Quin̄ones Km. 2.5. Apartado Postal 784. Teléfonos: 26-5515, 26-5516. Telefax: 26-5527. E-mail: dirpea@iiap.org.pe

2 Dirección de Extracción y Procesamiento Pesquero. Dirección Regional de la Producción de Loreto (PRODUCE Loreto). Av. Ramírez Hurtado c/. Jirón San Martín. Telálono: 23-2202. Iquitos, Perú

3 Dirección de Acuicultura. Dirección Regional de la Producción de Loreto (PRODUCE Loreto). Av. Ramírez Hurtado c/. Jirón San Martín. Teléfono: 232202. Iquitos, Perú

4 ONG Pro Naturaleza. Programa Regional Loreto. Calle Bermúdez 791. Iquitos. Teléfono; 60-0472. E-mail: jnoriega@pronaturaleza.org

- Autor para correspondencia: Calle Arica 508, lquitos, Loreto, Perú. Celular(065) 993-1025. E-mail: alcanf@iiap.org.pe 


\section{INTRODUCCIÓN}

La arahuana Osteoglossum bicirrhosum es un pez de origen amazónico que vive en ambientes de agua negra, ligeramente ácida, con alto contenido de material húmico derivado de la descomposición de la materia orgánica de origen vegetal, ya sea del entomo del ambiente acuático, o de las macrófitas flotantes, propias de estos ecosistemas.

$\mathrm{La}$ arahuana pertenece al orden Osteoglossiformes, familia Osteoglossidae y subfamilia Osteoglossinae por poseer una lengua ósea. Los dos miembros de la familia Osteoglossidae $(O$. bicirrhosum y $O$. ferreirae) están distribuidos en toda la cuenca amazónica y parte de la cuenca de los ríos Negro y Orinoco en Sudamérica (Nelson, 1979; Rabello, 1999; Sánchez y Vásquez, 2005). Las dos especies de arahuanas sudamericanas existentes, vienen siendo intensamente explotadas, al punto que en Colombia, ambas especies figuran en el Libro Rojo de las especies icticas colombianas (Mojica et al. 2002; Argumedo, 2005).

Este pez se caracteriza por poseer un cuerpo alargado, grande y comprimido de un color plateado grisáceo, cubierto de escamas cicloideas grandes. La cabeza es grande, típica de un pez carnivoro, provista de una boca grande, oblicua, con dientes caniniformes, con presencia de dos barbillas en el mentón, característica de la familia Osteoglossidae y que, a la vez, lo diferencia de la familia Arapaimidae (Powell, 1998). Presenta aletas pélvicas sin espinas y aletas dorsal y anal alargadas.

Algunos estudios enfocados en la crianza en cautiverio, biologia reproductiva y el aprovechamiento sostenido de la especie fueron realizados en la Amazonia Peruana, especificamente en el ámbito de la Reserva Nacional Pacaya Samiria (Alcántara, 1980; Tang y Gómez, 2005). En este artículo se presenta un análisis sobre las zonas y épocas de pesca, los volúmenes de comercialización y las posibilidades del cultivo de la arahuana, en Loreto, Perú.

\section{MATERIAL Y MÉTODO}

En el presente artículo se analiza la información sobre niveles de desembarque de crias de arahuana Osteoglossum bicirrhosum entre los años 1989 y 1990 y datos de la comercialización de esta especie entre los años 1999 a 2007 en la región Loreto (Perú) procedente de los registros de la Dirección de Extracción y Procesamiento Pesquero de la Dirección Regional de la Producción de Loreto, del Instituto Nacional de Recursos Naturales (INRENA) y de la ONG Pro Naturaleza, entidades con sede en la ciudad de Iquitos. Adicionalmente, informaciones relevantes sobre niveles de desembarque fue proporcionada por el ex Director de la Dirección Regional de Pesquería, el Sr. Santos Rubiños y niveles de comercialización fue proporcionada por el Sr. Edgar Panduro de la empresa Ornamental Amazon Fish Aquarium (OAFA). A su vez, la información correspondiente a cuotas de pesca de las unidades de pesca comunitaria de la Reserva Nacional Pacaya Samiria fue proporcionada por Cristian Tanchiva de INRENA y personal de la ONG conservacionista Pro Naturaleza.

\section{RESULTADOS}

\section{ANÁLISIS DEL DESEMBARQUE DEARAHUANA ENTRE 1989-1990.}

El desembarque de crías de arahuana procedente de los sistemas de los ríos Ucayali y Amazonas (Loreto, Perú) es estacional y cubre el periodo comprendido entre octubre y marzo, con un máximo en intensidad en enero, época en que la especie se reproduce en el sistema del río Ucayali. La reproducción de esta especie es periódica y comprendida entre los meses de Octubre a Marzo de cada año en relación directa con la época de expansión del ambiente acuático, como ya fue reportado por Dos Santos et al. (1984) en Brasil.

En la temporada de pesca comprendida entre 1989 y 1990 operaron, en Iquitos, 18 acuarios comerciales que reportaron un nivel de desembarque de 298,666 larvas (Tabla 1, Figura 1).

En el periodo mencionado, se declaró nueve zonas de pesca de arahuana: rio Ucayali, Canal del Puinahua, río Tapiche, río Blanco, río Amazonas, río Nanay, río Napo, rio Tigre y rio Maniti, siendo la más importante, por el nivel de captura, el rio Ucayali con cerca del $66 \%$ de los desembarques (Tabla 2, Figura 2).

Dieciocho empresas comerciales reportaron desembarques de arahuana en el período 1989 - 1990 $y$ las que reportaron el mayor desembarque en Iquitos fucron Bluc Acuario S. C. R. L. y Acuario C \& J con 50,000 y 38,000 larvas cada una, respectivamente, superando de lejos a las demás empresas (Figura 3).

\section{ANÁLISIS DELA COMERCIALIZACIÓN DELA}

\section{ARAHUANA ENTRE $1999-2007$}

En el período comprendido entre los años 1999 al 2007 se observa un nivel promedio de comercialización de larvas de arahuana en Iquitos, de aproximadamente 840,914 larvas por año, por un valor de 707,552 dólares americanos (Tabla 3 ).

Relacionando los desembarques procedentes del río Ucayali con el total desembarcado en el periodo comprendido entre octubre de 1989 y 
Tabla 1. Desembarque de arahuana 0 . bicirrhosum en la ciudad de lquitos entre los años 1989- 1990.

\begin{tabular}{|c|c|c|c|c|c|c|c|c|c|c|}
\hline \multirow[b]{2}{*}{$\mathbf{N}^{0}$} & \multirow[t]{2}{*}{ ACUARIO } & \multicolumn{4}{|c|}{1889} & \multicolumn{4}{|c|}{1990} & \multirow{2}{*}{$\begin{array}{l}\text { TOTAL } \\
\text { GENERAL }\end{array}$} \\
\hline & & Oct & Nov & Dic & Total & Ene & Feb & Mar & Total & \\
\hline 1 & $C \& J$ & 0 & 2,420 & 17,850 & 20,270 & 7,950 & 4,810 & 3,988 & 16,748 & 37,018 \\
\hline 2 & Cria Pez S.C.R.L & 0 & 4,000 & 14,950 & 18,950 & 4,400 & 0 & 0 & 4,400 & 23,350 \\
\hline 3 & Itaya & 300 & 0 & 2,800 & 3,100 & 0 & 0 & 0 & 0 & 3,100 \\
\hline 4 & Luis Dávila P. & 0 & 80 & 2,440 & 2,520 & 2,960 & 660 & 1,080 & 4,700 & 7,220 \\
\hline 5 & Sur América & 0 & 350 & 11,100 & 11,450 & 800 & 0 & 0 & 800 & 12,250 \\
\hline 6 & Aberto Coriat & 0 & 0 & 17,805 & 17,805 & 0 & 0 & 0 & 0 & 17,805 \\
\hline 7 & Blue Acuario S.C.R.L & 365 & 3,400 & 20,100 & 23,865 & 10,360 & 7,650 & 6,000 & 24,010 & 47,875 \\
\hline 8 & Chullachaqui & 143 & 2,580 & 3,670 & 6,393 & 0 & 1,010 & 65 & 1,075 & 7,468 \\
\hline 9 & Dolphin Peru Ltda. & 6,660 & 0 & 4,500 & 11,160 & 0 & 0 & 0 & 0 & 11,160 \\
\hline 10 & Edgar Bustamante & 0 & 0 & 5,000 & 5,000 & 80 & 70 & 0 & 150 & 5,150 \\
\hline 11 & Iquitos Fishery Ac. & 0 & 11,100 & 12,803 & 23,903 & 0 & 0 & 0 & 0 & 23,903 \\
\hline 12 & José Saénz Manzur & 0 & 2,250 & 6,500 & 8,750 & 1,000 & 500 & 0 & 1,500 & 10,250 \\
\hline 13 & Marcos Novoa & 12 & 7,500 & 5,200 & 12,712 & 0 & 0 & 0 & 0 & 12,712 \\
\hline 14 & Peces de la Amaz. & 0 & 1,500 & 14,900 & 16,400 & 2,000 & 800 & 500 & 3,300 & 19,700 \\
\hline 15 & Peru Fish S.C.R.L & 210 & 510 & 6,600 & 7,320 & 0 & 0 & 0 & 0 & 7,320 \\
\hline 16 & Piscis Aquarium & 0 & 130 & 5,550 & 5,680 & 0 & 0 & 0 & 0 & 5,680 \\
\hline 17 & Tropical E.I.R.L & 1,500 & 3,775 & 16,100 & 21,375 & 1,600 & 120 & 5,000 & 6,720 & 28,095 \\
\hline \multirow[t]{2}{*}{18} & Yacuruna S.C.R.L & 6,220 & 3,600 & 6,600 & 16,420 & 0 & 780 & 1,410 & 2,190 & 18,610 \\
\hline & TOTAL. & 15,410 & 43,195 & 174,468 & 233,073 & 31,150 & 16,400 & 18,043 & 65,593 & 298,666 \\
\hline
\end{tabular}

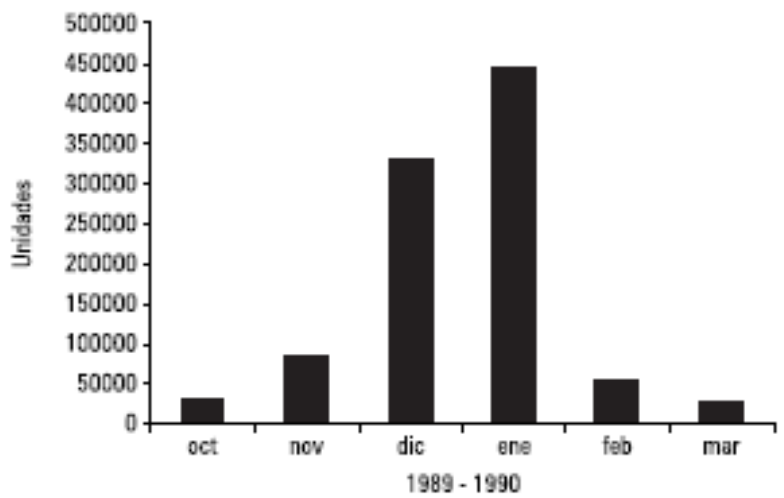

Figura 1. Variación temporal del desembarque de arahuana 0 . bicirrhosum en Iquitos entre los años 1989-1990.

marzo de 1990 , se tiene que el $67 \%$ de larvas procede del sistema del río Ucayali, en el cual se encuentra comprendida la Reserva Nacional Pacaya Samiria (RNPS), por lo que se asume que parte del desembarque declarado procede de dicha área. Este supuesto se sustenta en el hecho de que, con el correr de los años, han sido establecidas cuotas para los grupos organizados denominados Unidades de Pesca Comunitaria (UPC) que operan en el ámbito de esta reserva nacional, con autorización del Instituto Nacional de Recursos Naturales - INRENA y de la Dirección Regional de la Producción de Loreto, que se encargan de la administración y de los aspectos técnico normativos, correspondientes.

En el año 2006 operaron en el ámbito de la RNPS doce UPC, en su mayoría ubicadas en las localidades de Manco Capac, Bretaña y Yarina. Estas contaron con cuotas de pesca previamente establecidas por las entidades normativas y ejercieron una captura de cerca de 80,000 larvas de arahuana, por un valor de venta aproximado de más de 80,000 dólares americanos (Tabla 4).

Si en el período 1989 - 1990 operaron, en Iquitos, 18 empresas de exportación de peces ornamentales; en el 2005 operaron 35 empresas (Anónimo, 2005), con un crecimiento de $100 \%$, lo que evidenció una tendencia creciente en la instalación y operación de empresas de comercialización de peces ornamentales 
FOUA

LA PESQUERIA ORNAMENTAL DE LA ARAHUANA Ostooglossum bicinthosum

Amazónico 1 (OSTEOGLOSSIDAE) EN LORETO, PERÚ Y POSIBUIDADES DE SU CULTVOO

Tabla 2. Zonas de pesca de arahuana 0 . bicirnosum en la región Loreto entre los años 1989-1990.

\begin{tabular}{|c|c|c|c|c|c|c|c|c|c|c|}
\hline MES & $\begin{array}{c}\text { Rio } \\
\text { Ucayali }\end{array}$ & $\begin{array}{c}\text { Canal } \\
\text { Puinahua }\end{array}$ & $\begin{array}{c}\text { Río } \\
\text { Tapiche }\end{array}$ & $\begin{array}{c}\text { Río } \\
\text { Blanco }\end{array}$ & $\begin{array}{c}\text { Río } \\
\text { Amazonas }\end{array}$ & $\begin{array}{c}\text { Rio } \\
\text { Nanay }\end{array}$ & $\begin{array}{c}\text { Río } \\
\text { Napo }\end{array}$ & $\begin{array}{l}\text { Rio } \\
\text { Tigre }\end{array}$ & $\begin{array}{c}\text { Rio } \\
\text { Manití }\end{array}$ & TOTAL \\
\hline Octubre '89 & 7,985 & 6,500 & 143 & 610 & 0 & 12 & & & & 15,250 \\
\hline Noviembre ' 89 & 51,770 & 800 & 1,280 & 1,980 & 1,980 & 8,595 & & & & 68,595 \\
\hline Diciembre '89 & 92,405 & & 28,100 & 16,000 & 4,670 & 2,540 & 553 & 1,100 & & 145,368 \\
\hline Enero '90 & 26,710 & & 2,860 & 1,600 & 1,120 & 2,640 & & & 80 & 35,010 \\
\hline Febrero '90 & 10,950 & & 3,870 & 0 & 1,010 & 500 & & & 70 & 16,400 \\
\hline Marzo '90 & 9,130 & & 8,768 & 0 & 65 & 80 & & & & 18,043 \\
\hline TOTAL & 198,950 & 7,300 & 45,021 & 22,380 & 8,845 & 14,367 & 553 & 1,100 & 150 & 298,666 \\
\hline
\end{tabular}

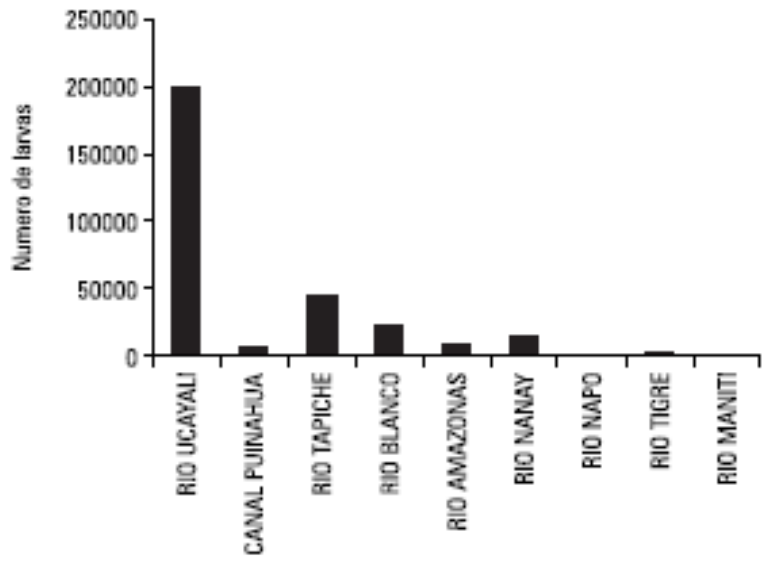

Figura 2. Larvas de arahuana desembarcadas en lquitos por zona de pesca. $1989-1990$. durante es período, en Iquitos. Sin embargo, el número de larvas comercializadas se ha mantenido más o menos estable en el período 1999 a 2005 lo que, aparentemente ha determinado el incremento de los precios de las larvas en el mercado de Iquitos.

Entre los años 2006 y 2007 solo 11 empresas exportaron crías de arahuana de la región Loreto: Dragom Fish Farm Trading E.I.R.L., Stingray Aquarium S.A.C., Ornamental Amazon Fish Aquarium S.A.C., Amazon Tropicals Aquarium E.I.R.L., Agafex, Fapex E.I.R.L., Sur America S.R.L., Aquarium Río Momón E.I.R.L., Myriam I, Peru Aquarium Group S.A.C. y Nijsseni's Corporation S.R.L., empero, los niveles de extracción y exportación de crías aumentó en más de 145,000 unidades entre el 2005 y el 2007.

Comparando el nivel de desembarque de crias de arahuana del periodo 1989-1990, con el de comercialización registrado en el 2007 , se deduce un claro incremento en la presión de pesca del recurso en

Tabla 3. Larvas de arahuana 0. bicirrhosum exportadas al extranjero desde Loreto (Perú) en los últimos nueve años (1999 -2007).

\begin{tabular}{c|c|cc}
\hline Año & $\begin{array}{c}\text { Unidades } \\
\text { Comercializadas }\end{array}$ & $\begin{array}{c}\text { Valor } \\
\text { (En dólares USA) }\end{array}$ & $\begin{array}{c}\text { Precio promedio } \\
\text { (En dólares USA) }\end{array}$ \\
\hline $1999^{\prime}$ & 749,907 & $310,999.21$ & 0.414 \\
\hline $2000^{\prime}$ & 717,425 & $309,067.28$ & 0.430 \\
\hline $2001^{\prime}$ & $1,007,460$ & $559,614.75$ & 0.555 \\
\hline $2002^{\prime}$ & 742,018 & $559,614.75$ & 0.754 \\
\hline $2003^{\prime}$ & $1,200,785$ & $1,113,701.68$ & 0.927 \\
\hline $2004^{\prime}$ & 770,479 & $632,747.78$ & 0.821 \\
\hline $2005^{\prime}$ & 708,613 & $740,768.35$ & 1.045 \\
\hline $2006^{2}$ & 816,872 & $1,004,752.56$ & 1.230 \\
\hline $2007^{2}$ & 854,666 & $1,136,705.78$ & 1.330 \\
\hline Total $^{2}$ & $7,568,225$ & $6,367,972$ & $\ldots$ \\
\hline
\end{tabular}




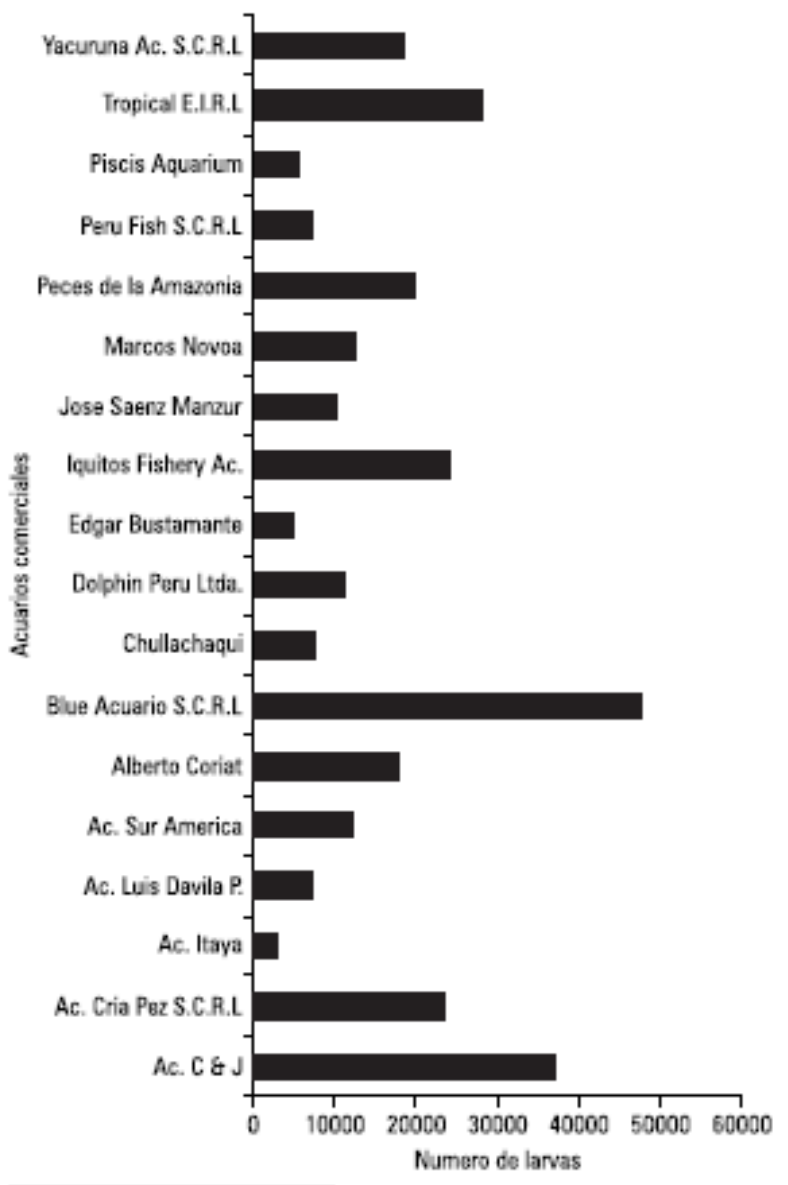

Figura 3. Larvas de arahuana 0 . bicirrhosum desembarcadas por acuario en la ciudad de lquitos entre los años $1989-1990$. la región Loreto, especialmente en el sistema del Río Ucayali, y en el ámbito de la RNPS, incrementándose desde cerca de 300,000 unidades, en 1990 a 854,666 , en el 2007, que se debe, a la mayor demanda del mercado, al incremento del precio unitario, como a la disponibilidad y accesibilidad a la captura en el ámbito de la RNPS, observada durante los últimos años.

\section{DISCUSIÓN}

La reproducción de la arahuana generalmente ocurre durante la subida de las aguas, de diciembre a marzo y los progenitores proporcionan cuidados a la prole, alojando a los jóvenes en la cavidad bucal en caso de peligro, como lo reportaron Dos Santos et al. (1984, op cit.). Las larvas presentan un saco vitelino bastante grande y de color rojo naranja intenso que, alcanza un peso mayor al peso del cuerpo y les da un atractivo particular.

Por las características antes señaladas la arahuana es un pez altamente cotizado en el mercado internacional, en especial, asiático, por que se la relaciona con el dragón y determina un flujo de varios cientos de miles de dólares anuales para el Perú, por su exportación. Sin embargo, el comercio se sustenta, exclusivamente, de la extracción de larvas y alevinos del medio natural capturando los adultos con diversos aparejos, desde redes y balas, con una captura anual creciente que comercializan varias empresas localizadas en Iquitos.

Asimismo, tenemos que considerar que el número de crías que se exporta anualmente es en realidad solo

Tabla 4. Cuotas y captura de larvas de arahuana 0. bicirrhosum por Unidad de Pesca Comunitaria, por comunidad y por cuenca. Fuente: INRENA (C. Tanchiva. 2006)

\begin{tabular}{c|l|l|c|c|c|}
\hline$N^{0}$ & Grupo & Comunidad & $\begin{array}{c}\text { Cuota } \\
\text { asignada }\end{array}$ & Captura & Cuenca \\
\hline 1 & Lobitos & Manco Cápac & 5,603 & 8,003 & Pacaya \\
\hline 2 & Leones & Bretaña & 10,888 & 12,479 & Pacaya \\
\hline 3 & Martin Pescador & Bretaña & 2,153 & 2,722 & Pacaya \\
\hline 4 & Zungaritos & Bretaña & 2,266 & 6,924 & Pacaya \\
\hline 5 & Tucanes & Bretaña & 7,527 & 10,370 & Pacaya \\
\hline 6 & Guacamayos & Bretaña & 9,032 & 5,050 & Pacaya \\
\hline 7 & Catalanes & Bretaña & 9,732 & 5,066 & Pacaya \\
\hline 8 & Tibes & Bretaña & 6,297 & 0 & Pacaya \\
\hline 9 & Lobos & Bretaña & 6,707 & 8,170 & Pacaya \\
\hline 10 & UPC Yarina & Yarina & 3,505 & 2,880 & Yanayacu Pucate \\
\hline 11 & Tibes & Manco Capac & 7,199 & 5,242 & Yanayacu Pucate \\
\hline 12 & Yacutaìta & Manco Capac & 14,248 & 12,135 & Yanayacu Pucate \\
\hline Total & & & 85,157 & 79,041 & \\
\hline
\end{tabular}


un porcentaje de lo que se extrae del medio natural al cual hay que sumar los peces que se pierden desde el momento de la extracción, pasando por el manipuleo propio del transporte a Iquitos y el posterior manejo en manos de los acopiadores y de los exportadores. En el mismo sentido, los 6'367,972 dólares estadounidenses que se estima ha sido el valor en divisas derivados de la exportación de este recurso en los últimos nueve años probablemente sea una cifra mucho menor a los montos que se manejan en la realidad.

Desde cualquier punto de vista, la extracción de crías arahuana en la región Loreto es una actividad insostenible en el tiempo, debido a que generalmente se sacrifican los machos adultos que brindan el cuidado parental a las crías para de ese modo obtener las larvas, afectando negativamente la proporción sexual en las poblaciones naturales de esta especie. Algunos acuaristas locales sospechan que en la actualidad, la proporción sexual en las poblaciones de arahuana de la RNPS es de un macho por cada cuatro hembras (Edgar Panduro, entrevista personal). Adicionalmente, expertos del IRD (Francia) y del IIAP (Perú) vienen evaluando el recurso arahuana y estiman que el sex ratio de las poblaciones naturales continuará en deterioro por la pesca indiscriminada de ejemplares machos. En ese sentido, de continuarse utilizando los mismos métodos de extracción de crías, se estaría comprometiendo el futuro mismo de la especie, sobre todo en las zonas libres del control del Estado como las zonas de pesca localizadas fuera del ámbito de la RNPS (Putumayo, Tapiche, Ucayali, etc.).

\section{EL CULTIVO DE LA ARAHUANA COMO}

\section{ALTERNATIVA}

$\mathrm{La}$ arahuana se reproduce naturalmente en estanques, como lo observó Alcántara (1980) en el Laboratorio de Iquitos del IMARPE, en Quistococha, comprobando la producción de 100 a 300 crías por hembra (promedio de 220 crías según Argumedo 2005), por año, por lo cual, resulta técnicamente factible, la producción de crías de esta especie en piscicultura para así mejorar las condiciones de oferta y de ingresos del poblador regional. La demanda creciente de larvas del mercado, el buen precio de las larvas alcanzado durante los últimos años y la reproducción natural de la especie, en ambientes lénticos y estanques de cultivo, permite identificar la posibilidad de impulsar programas de cultivo y reproducción de esta importante especie para generar alternativas de desarrollo en la región amazónica que, de paso, requiere de nuevos enfoques dirigidos a promover el desarrollo sostenido, en función de la oferta ambiental.

El Instituto de Investigaciones de la Amazonía
Peruana (IIAP) y al menos un empresario privado del sector exportador de peces ornamentales de la ciudad de Iquitos están dando los primeros pasos para establecer las bases científicas y tecnológicas que conlleven a la consecución del paquete tecnológico del cultivo de esta especie en cautiverio y que de ese modo asegure en el mediano plazo el abastecimiento de crías de arahuana provenientes de acuicultura. Entre los años 2008 y 2010, el IIAP contando con el apoyo científico del IRD francés y la ayuda económica del Proyecto INCAGRO, desarrollará una serie de estudios (rasgos de vida, estructura genética, reproducción inducida, piscicultura y manejo de crías en condiciones controladas) con la finalidad de contribuir en el manejo sostenible de la especie y garantizar su conservación en los ecosistemas acuáticos de la región Loreto.

Por las consideraciones antes señaladas indicamos como recomendaciones: i) evitar en la medida de lo posible el sacrificio de los ejemplares adultos durante la pesca de las crías, ii) que la extracción de arahuana en la región Loreto sea regulada y permitida solo a los grupos organizados que cuenten con planes de manejo coherentes, sin perjuicio de su ubicación geográfica, determinándose una cuota anual de extracción tal como se hace en las UPC de la RNPS, iii) que las cuotas de comercialización señaladas para las empresas de exportación sean cotejadas con las cuotas de extracción de los grupos de manejo pesquero para prevenir excesos iv) propiciar el establecimiento de Unidades de Pesca Comunitaria (UPC) en todas las zonas de extracción de arahuana, v) procesar la información de extracción y comercialización de arahuana con eficiencia y rapidez para evitar la exportación de peces sobre las cuotas de extracción establecidas y vi) promover estudios de crianza, reproducción y manejo de crías de arahuana en ambientes controlados.

\section{CONCLUSIONES}

Las principales zonas de extracción de crías de arahuana con fines ornamentales son la Reserva Nacional Pacaya - Samiria, el sistema del Ucayali Tapiche y parte de la cuenca del río Putumayo.

El período de pesca de las crías de arahuana coincide con la época de reproducción natural de la especie siendo más frecuente entre los meses de diciembre y marzo en la región Loreto.

De acuerdo a las estadisticas analizadas, $7^{\prime} 568,225$ crías de arahuana han sido exportadas en los últimos nueve años desde la región Loreto, lo que hace un promedio de 840,914 crías por año y un total de 6‘367,972 dólares estadounidenses.

El cultivo y la producción de crías de arahuana en 
cautiverio (piscicultura) es una alternativa real para contribuir al manejo sostenible de esta actividad y a la conservación de la especie.

\section{AGRADECIMIENTOS}

Los autores expresan su especial agradecimiento a los señores Santos Rubiños y Edgar Panduro y al biólogo Cristian Tanchiva, Javier Noriega y Jorge Gómez, quienes proporcionaron gran parte de la información que se analiza en este documento.

\section{REFERENCIAS BIBLIOGRÁFICAS}

ALCÁNTARA BF. Informe anual de actividades. Instituto del Mar del Perú. Laboratorio de Iquitos. Informe interno. 5 pp. 1980.

ANÓNIMO. Catálogo de Peces Ornamentales. IIAP. PROMPEX. 45 pp. 2005.

ARGUMEDOTEG. Arawanas. Manual para la cría comercial en cautiverio. Asociación de Acuicultores del Caquetá (ACUICA) y Fondo para la Acción Ambiental. Edit. PRODUMEDIOS, Bogota, 105 pp. 2005.

DOS SANTOS GM, JEGU M, DE MERONA B. Catalogo de peixes comerciais do Baixo Rio Tocantins. Centrais Eletricas Do Norte Do Brasil S. A. - Eletronorte. Conselho Nacional de Desenvolvimento Cientifico e Tecnologico CNPq. Instituto Nacional de Pesquisas Da Amazonia - INPA. Projeto Tucuruí. $1^{\mathrm{a}}$ edicao. 20 pp. 1984.
MOJICA JI, CASTELLANOS C, USMA S, ALVAREZ R. Libro Rojo de Peces Dulceacuícolas de Colombia. Serie: Libros Rojos de Especies Amenazadas de Colombia. Instituto de Ciencias Naturales, Universidad Nacional de Colombia, Ministerio del Medio Ambiente. Bogotá, Colombia. 2002.

MONTREUIL VH. Sistematización y análisis del manejo de arahuana en la Reserva Nacional Pacaya Samiria. Informe de consultoría. Pro Naturaleza. Programa Nor Este. Iquitos, Peru. 51 pp. 2006.

POWELL R. A Key to the Market Fishes of the Iquitos Region, Perú. Master of Science Thesis. Southern Illinois University at Carbondale. USA. $141 \mathrm{pp}$. 1998.

RABELLO J. Biología reprodutiva e alimentaçao do arahuana preto, Osteoglossum ferreirae, Kanazawa 1996 (Pisces, Osteoglossiformes) no Igarape Zamula, medio rio Negro, AM, Brasil. Tesis de Ingeniero de Pesca. Universidade do Amazonas, Brasil. 1999.

SÁNCHEZ RH, VÁSQUEZ J. Peruvian Ornamental Fish. IIAP. PROMPEX. Primera Edición. 36 pp. 2005.

TANG M, GÓMEZ J. Biología y aprovechamiento de la arahuana Osteoglossum bicirrhosum en la micro cuenca de la cocha el Dorado: Reserva Nacional Pacaya Samiria. Tesis para optar Título de Biólogo. Universidad Nacional de la Amazonía Peruana. Iquitos, Perú. 121 pp. 2005. 